The influence of attention on mathematical knowledge of teachers and lecturers: A comparison

Sergiy Klymchuk ${ }^{*}$ and Mike Thomas ${ }^{* *}$

*School of Computing and Mathematical Sciences, Auckland University of Technology, Auckland, New Zealand

**Department of Mathematics, The University of Auckland, Auckland, New Zealand

Corresponding author:

Dr Sergiy Klymchuk

Associate Professor

School of Computing and Mathematical Sciences

Faculty of Design and Creative Technologies

Auckland University of Technology

Private Bag 92006

Auckland 1142

New Zealand

Tel: +64-9-921 9999 ext.8431

Fax: +64-9-921 9944

E-mail: sergiy.klymchuk@aut.ac.nz 


\title{
The influence of attention on mathematical knowledge of teachers and lecturers: A comparison
}

\begin{abstract}
This paper reports on some findings from the project 'Analysing the Transition from Secondary to Tertiary Education in Mathematics'. One of key variables in the school to university transition is the teacher/lecturer, and here we deal with data analysing secondary teachers' and tertiary lecturers' responses to four mathematics questions. Elsewhere we consider knowledge, preparedness and teaching style etc, but this paper tracks the ability to use mathematical procedures. We hypothesise that this is a function of what we pay attention to, as described in Mason's discipline of noticing. The results reveal that many teachers and lecturers fail to notice the necessary conditions for problems that imply that procedures are not always applicable. Possible reasons for this along with implications for student learning are discussed.
\end{abstract}

Keywords: attention; mathematical knowledge; teachers; lecturers

\section{Introduction and framework}

The transition from school to university is coming under increased scrutiny in the light of growing concerns about decreasing numbers of students opting to study mathematics at university and beyond (e.g., the ICMI Pipeline Project), and their apparently decreasing levels of competence [1]. While the increasing numbers and diversity of those attending higher education institutions are an issue, a lack of essential technical facility, a decline in analytical powers and a lack of appreciation of the place of proof in mathematics on the part of undergraduates have been cited in the USA and UK $[2,1]$. The importance of this transition period is also increasingly reflected in research papers and international forums (e.g. $[3,4]$ ). One possible reason for such transition problems may be in the mathematical emphasis at each level. A developing theory by Tall [4] suggests that mathematical thinking exists in three 'worlds': an embodied world where we make use of physical attributes of concepts, combined with our sensual experiences to build mental conceptions; a symbolic world where the symbolic representations of concepts are acted upon, or manipulated; and the formal world where properties of objects are formalized as axioms, and learning comprises the building and proving of theorems by logical deduction from axioms. There is evidence that school mathematics draws heavily on the symbolic world while in university the emphasis is more on the formal world $[5,6]$. Thus both schoolteacher and lecturer may need to draw on, and integrate, more than one kind of thinking. The scope and nature of the mathematical knowledge needed for teaching has been a focus of research by Deborah Ball and her colleagues, who have described in detail the concept of mathematical knowledge for teaching $[7,8]$, which extends Shulman's pedagogical content knowledge, combining three kinds of subject matter knowledge with three kinds of pedagogical knowledge. One use of this knowledge for teaching is described as: 'Teachers do not merely do problems while students watch... They must choose useful models or examples. Doing these things requires additional mathematical insight and understanding' [7, p. 17]. One of the aspects of this paper is devoted to the teacher's and lecturer's ability to choose suitable problems for calculus students and the insight needed to do so. The other aspect of the paper deals with the teacher's and lecturer's ability to pay attention to conditions of problems before applying a certain rule or technique.

The theoretical framework of the paper is based on research by John Mason [9,10]. Mason has proposed that when we look at mathematics the focus of our attention may vary depending on whether we are looking at the symbols or looking through them. The idea is that we need to structure our attention, to know what we are aware of, and Mason describes a 
number of elements that we may focus attention on, including: the whole, the details, the relationships between the parts, the properties of the whole or the parts and deductions [9]. One way in which a teacher may focus student attention is by asking questions [10]. The style of these questions, which will often be oral, but may include written questions, is crucial if attention is to be focussed on what is to be learned. As Mason says "...the style and format of the questions used by lecturers and tutors profoundly influence students' conceptions of what mathematics is about and how it is conducted. By looking at reasons for asking questions, and becoming aware of different types of questions which mathematicians typically ask themselves, we can enrich students' experience of mathematics." [10, p. 97]. However, in spite of the best efforts of teacher or lecturer when they ask questions there can be a mismatch of attention, caused by teacher and students attending to different things from among: holistic ideas; details; relationships among details; properties of objects in general; or deductions from object properties [9, p. 9]. Such a process involves aspects of knowing-about that Mason and Spence [11] describe, namely knowing-that (factual), knowing-how (techniques and skills), or knowing-why (ability to restructure actions). We are confident that the vast majority of teachers and lecturers who teach calculus have excellent knowing-that and knowing-how mathematics knowledge. Hence the question is whether this kind of knowledge can structure attention in such a way that a procedural emphasis tends to develop, thus obscuring detail it may be crucial to notice.

\section{The study}

This study is part of a much larger research project entitled 'Analysing the Transition from Secondary to Tertiary Education in Mathematics', involving teachers, lecturers and students, that employs questionnaires, interviews and observations. Questionnaires were sent to all 350 secondary schools in New Zealand to be completed by all teachers who teach calculus in Years 12 or 13 (age 17-18 years) and to the 31 Tertiary Institutions (Polytechnics, Universities and Institutes of Technology) in New Zealand to be completed by all the lecturers who teach calculus or teach in courses with calculus components. The questionnaires were posted, complete with a stamped addressed return envelope and potential respondents were given three weeks to answer. After this a follow-up copy was sent by email to remind the teachers and lecturers to reply. Using this approach we received a total of 178 teacher and 25 lecturer responses. Since there are no figures available on the total number of calculus teachers in New Zealand schools, which vary in size from fewer than 30 students (small country school) to 3000 (inner city), we can only estimate the response rate at about $30 \%$ of that population. The response rate is similar with regard to tertiary lecturers.

In this paper we present and analyse teachers' and lecturers' responses to four related questions from the questionnaire. The first two questions were considered to be fairly routine assessment questions while the others looked like routine assessment questions but require solvers to notice that certain conditions need to be paid attention to before applying a standard procedure, rule or technique. The instruction given to the teachers was as follows: Please try the following questions and then comment on whether you think each of them is a suitable assessment item for a Year 13 [age 18 years] calculus class. A similar instruction was given to the lecturers:

Please try the following questions and then comment on whether you think each of them is a suitable assessment item for a first-year degree calculus class.

The four questions were:

Question 1. If $\int_{1}^{a}(2 x-1) d x=2$, find the possible values of $a$. 
Question 2. Sketch the graph of a function $f(x)$ such that it is continuous on $0 \leq x<3$ and $3<x \leq 5, f(3)=1$, and $f^{\prime}(x)>0$ for $0 \leq x \leq 5, x \neq 3$. Does $\lim _{x \rightarrow 3} f(x)$ exist for your function?

Question 3. Find the derivative of the function $y=\ln (2 \sin (3 x)-4)$.

Question 4. Find the integral $\int_{-1}^{1} \frac{1}{x} d x$.

Questions 1 and 2 were just routine test questions and were given to 'warm up' the participants. Question 3 looked like a routine question but had a difficulty because the function given for differentiation has an empty domain and hence is not differentiable. Question 4 might also look like a routine question to the teachers but it also had a potential problem for them - the integrand function has an infinite discontinuity at the point $x=0$ on the interval $[-1,1]$ and for that reason the integral in Question 4 is not a definite integral but an improper integral, which is beyond the school curriculum. For the lecturers Question 4 should have been just a routine test question on improper integrals. The main purpose was to check whether the participants of the study would pay attention to the conditions of Questions 3 and 4 and solve them correctly or go to the 'familiar' procedures and apply them in the situation where they were not applicable. Of course not all respondents attempted the questions or provided comments on them. In case of teachers the non-responses were often because the teacher had not taught Year 13 (age 18 years). Many respondents decided whether the questions were suitable for assessment without providing solutions to them. It was not possible to know whether they did any working, and if so whether it was correct. Therefore we concentrated more on those participants who provided their solutions to the four questions. Based solely on the school curriculum Questions 1, 2 and 3 are suitable to be considered for assessment items for a Year 13 calculus class and Question 4 is not. Based solely on the tertiary first-year degree calculus curriculum all four questions are probably suitable for assessment consideration. Tables 1 and 2 below summarise the teachers' and lecturers' responses to the four questions.

Table 1. A Summary of the Question Responses-Teachers.

\begin{tabular}{ccccccccc}
\hline Question & S & SC & SNC & NS & NSC & NSNC & $\begin{array}{c}\text { Not } \\
\text { Sure }\end{array}$ & $\begin{array}{c}\text { No } \\
\text { response }\end{array}$ \\
\hline 1 & 73 & 33 & 12 & 2 & 2 & 6 & 0 & 50 \\
2 & 68 & 31 & 2 & 15 & 6 & 2 & 0 & 54 \\
3 & 71 & 2 & 36 & 1 & 12 & 3 & 1 & 52 \\
4 & 42 & 3 & 22 & 23 & 23 & 9 & 2 & 54 \\
\hline
\end{tabular}

Table 2. A Summary of the Question Responses-Lecturers.

\begin{tabular}{ccccccccc}
\hline Question & S & SC & SNC & NS & NSC & NSNC & $\begin{array}{c}\text { Not } \\
\text { Sure }\end{array}$ & $\begin{array}{c}\text { No } \\
\text { response }\end{array}$ \\
\hline 1 & 14 & 7 & 4 & 0 & 0 & 0 & 0 & 0 \\
2 & 16 & 4 & 0 & 2 & 2 & 0 & 0 & 1 \\
3 & 15 & 1 & 7 & 0 & 1 & 1 & 0 & 0 \\
4 & 9 & 0 & 5 & 3 & 3 & 3 & 0 & 2 \\
\hline
\end{tabular}


Key: $\mathrm{S}=$ suitable for assessment no working; $\mathrm{SC}=$ suitable from correct working; $\mathrm{SNC}=$ suitable from incorrect working; NS=not suitable no working; $\mathrm{NSC}=$ not suitable from correct working; $\mathrm{NSNC}=$ not suitable from incorrect working.

\subsection{Questions 1 and 2 results}

Of the 128 teachers who responded to Question $192.2 \%$ thought it was a suitable assessment question, and 53 attempted to solve it with 35 giving a correct answer and 18 an incorrect one. Of these 18 some made calculation errors and some gave only the answer $a=2$, obtained 'by inspection' in one case. However, interestingly, 11 paid attention to the limits and rejected one of the solutions $a=-1$ on the grounds that it was negative. They all had the opinion that if a definite integral is positive then the upper limit is greater than the lower limit, and so, even though some had correctly calculated $a$ as negative, they then proceeded to reject it. It also appears that some found the actual concept of an integral $\int_{p}^{q} f(x) d x$ where $p>q$ difficult to accept. In the case of the teachers whose responses and explanations are shown in Figure 1, their mathematical belief has caused them to pay close attention to the sign of the solution for $a$, and hence to reject -1 as a solution. In doing so some also rejected the question as an assessment item, since it is 'too confusing', as the first teacher responds.
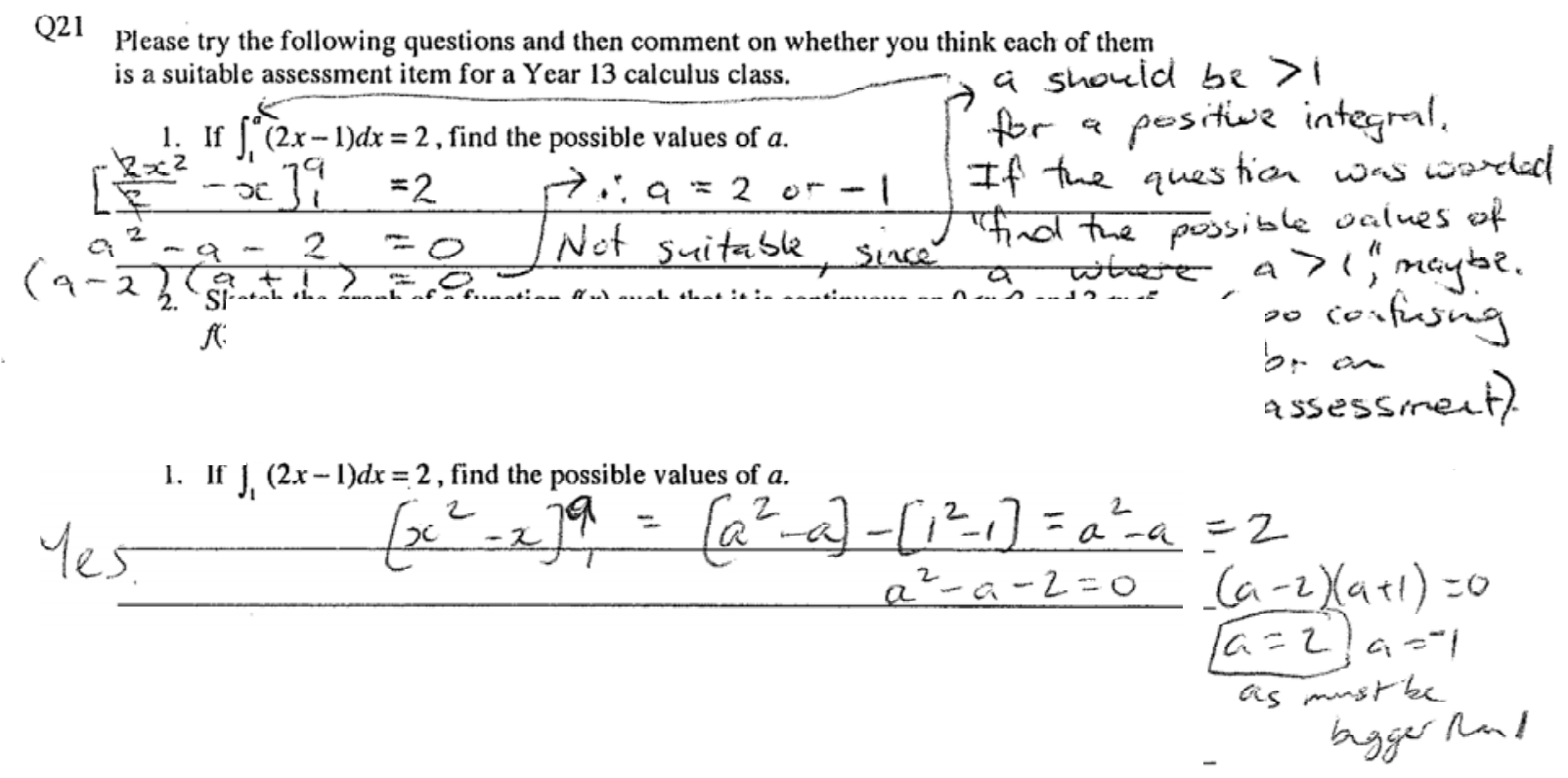

Figure 1. Responses of teachers to Question 1, rejecting one solution.

All 25 lecturers thought Question 1 was a suitable assessment question. Eleven lecturers provided solutions to Question 1 with seven correct working and four with incorrect working, rejecting -1 as a solution. Figure 2 below illustrates two incorrect lecturers' solutions to Question 1. 
1. If $\int_{1}^{a}(2 x-1) d x=2$, find the possible values of $a$.
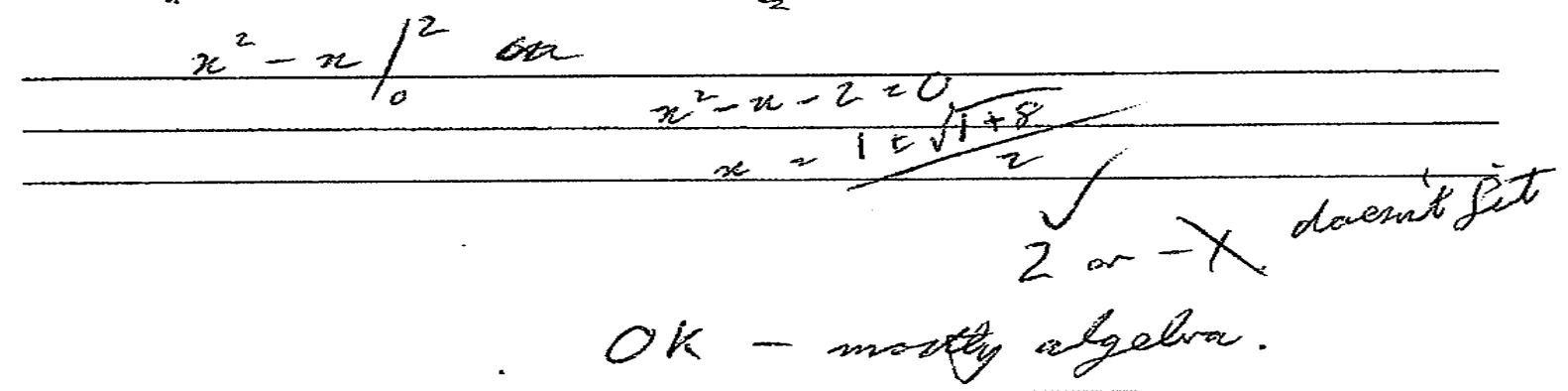

1. If $\int(2 x-1) d x=2$, find the possible values of $a$.

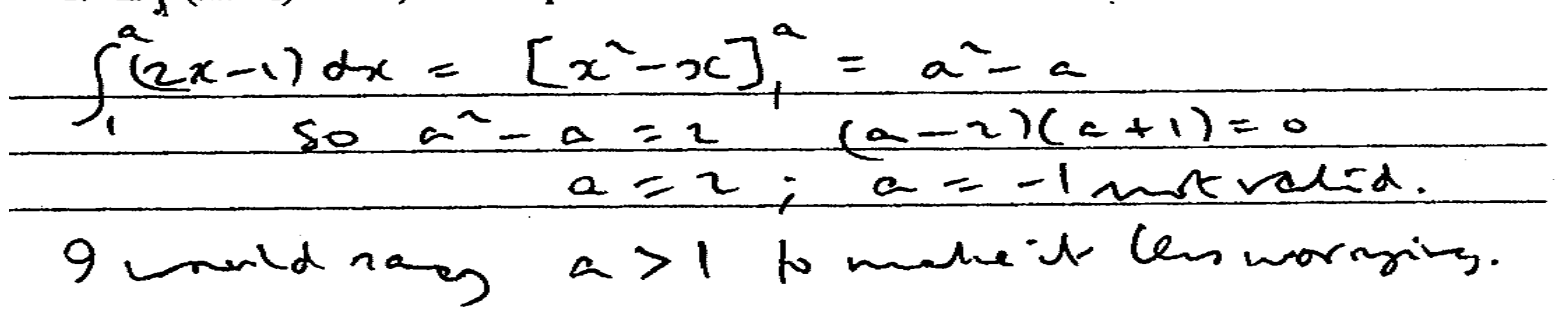

Figure 2. Responses of lecturers to Question 1, rejecting one solution.

We did not plan to try and 'catch out' the participants in Question 1 and were a little surprised to see that a number of the participants had a misconception about the definite integral, wrongly rejecting -1 as a solution. Another unexpected result was that the proportion of respondents who had that misconception was higher for the lecturers than for the teachers: $4 / 11$, or $36.4 \%$, for the lecturers versus $11 / 53$, or $20.8 \%$, for the teachers.

One hundred twenty four teachers responded to Question 2 with $101(81.5 \%)$ saying it was a good assessment question and 23 that it was not. Those who said yes commented that it 'requires some thought from students', 'tests students' understanding of the concepts of limits, continuity and differentiability', and 'it requires students to understand discontinuities', while those who answered no provided the comments like 'no perfect answer', 'open-ended', 'the concept of limits need not be this complicated' and 'attempting to draw the graph could confuse students'. Out of 24 lecturers who responded to Question 2 20 lecturers $(83.3 \%)$ said that it was a suitable question for assessment and four that it was not. So the preferences for including Question 2 in an assessment were very similar for the teachers and lecturers.

\subsection{Questions 3 and 4 results}

As mentioned above Questions 3 and 4 were the primary questions of interest in the study. Of the 125 teachers who responded to Question $3109(87.2 \%)$ thought was a suitable assessment question and 16 said it was not. Fifty three attempted to solve it with 14 giving a correct answer and 39 an incorrect one. Knowing the domain of the log function, and the range of the sine function should have been enough to solve it. Since calculus teachers can be expected to know the domain of the log function and the range of the sine function the question was not about their knowledge but about what they pay attention to, or notice. Yet, even though this is knowledge they have most of the teachers who attempted a solution failed to solve it (39/53 or $73.5 \%)$. The question is why? Question 3 had been deliberately set so that it was important to check the domain of the function and notice that the function is not defined because the argument of the log function is always negative. Hence the chain rule is not applicable in this case and the derivative does not exist. This may make it an unsuitable 
question in a primarily procedural assessment context. However, many teachers either 'solved' it using the standard technique, or wrote that the question was a 'good', 'straightforward', 'easy', or a 'suitable' question for assessment.

$$
\begin{aligned}
& \begin{aligned}
y^{\prime} & =\frac{1}{2 \ln (3 x)-2 \sin 3 x-4)^{\prime}} \\
& =\frac{2 \cos (3 x)(3 x)^{\prime}}{2 \sin (3 x)-4}=\frac{6 \cos (3 x)}{2 \sin (3 x)-4}=\frac{3 \cos (3 x)}{\sin (3 x)-2}
\end{aligned} \\
& y^{\prime}=\frac{1}{25(3 x)-4} \times 2 \cos (3 x) \times 3 \\
& \text { Yes. It is a good one to use of chein kate. }
\end{aligned}
$$

Figure 3. Two examples of incorrect teachers' responses to Question 3.

In Figure 3 we see two examples of solutions that apply the procedure without paying attention to the domain of the function, and one mentions that the question will 'reinforce use of the chain rule'. There is an implicit acceptance here that if the procedure 'works' and produces an answer then everything is fine. In contrast we see in Figure 4 examples of two teachers who, by paying attention to the domain, have noticed that there is a problem and hence reject it as a suitable assessment item. In the first case the procedure has been applied too, but in the second consideration of domain has been the first option.

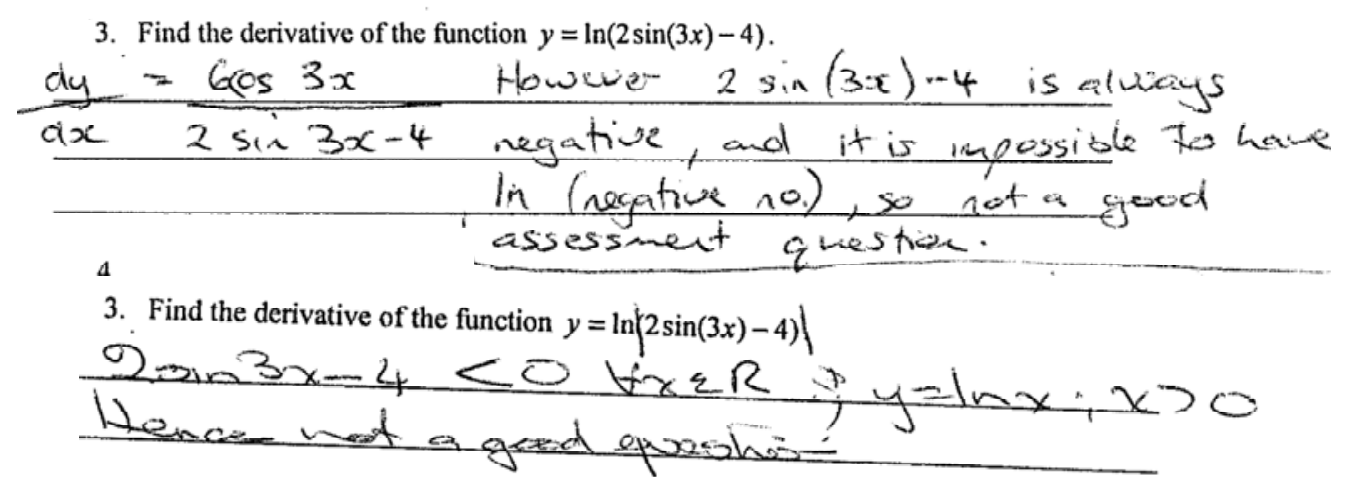

Figure 4. Teachers' responses to Question 3, showing recognition of the function domain.

Twenty five lecturers responded to Question 3 with 23 (92\%) saying it was a good assessment question (significantly more than the teachers, $\chi^{2}=9.12$ ) and two that it was not. Ten attempted to solve it, while two $(20 \%)$ gave a correct answer and eight $(80 \%)$ an incorrect one. So the proportion of incorrect solutions for Question 3 appeared slightly higher (but not significant, $\chi^{2}=0.18$ ) for the lecturers than for the teachers: $80 \%$ for the lecturers versus $73.5 \%$ for the teachers. We expected that the lecturers would be less 'procedure' oriented than the teachers, pay more attention to the properties of the functions involved, due to a habit of checking the conditions of a rule or a theorem before applying it. However, this was not the case for Question 3. Typical incorrect lecturers' solutions are presented in Figure 5. 
3. Find the derivative of the function $y=\ln (2 \sin (3 x)-4)$.

$\frac{d y}{d x}=\frac{1}{2 \sin (3 x)-4} \times(6 \cos 3 x)$

If only the first step is needed, this would be suitable at $3 \%: 1$ for each factor and 1 for the implementation of the Chain Rule.

3. Find the derivative of the function $y=\ln (2 \sin (3 x)-4)$.

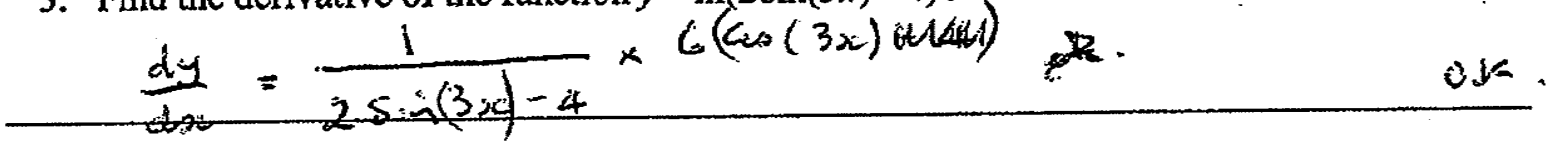

3. Find the derivative of the function $y=\ln (2 \sin (3 x)-4)$.

$\frac{d y}{d x}=\frac{6 \cos 3 x}{2 \sin 3 x-4}=\frac{3 \cos 3 x}{\sin 3 x-2}$

Figure 5. Three examples of incorrect lecturers' responses to Question 3.

Question 4 was the trickiest question of the survey for both groups - the teachers and lecturers. The integral in Question 4 is an improper integral (in this particular case it is undefined) and this topic is outside the school curriculum. What we expected from the teachers was a simple comment that the function $y=\frac{1}{x}$ is not continuous on the interval $[-1,1]$ (or not defined at the point $x=0$ ) and therefore the integral is not a definite integral and the Newton-Leibniz formula is not applicable. Again we may assume that while every teacher who teaches calculus knows this, what we pay attention to can influence actions. When there is a standard procedure that can be applied, does paying attention to this cause us to fail to notice other important detail such as the fact that the function is discontinuous on the interval and we come to an incorrect conclusion?

For Question 4, out of 122 teachers who responded 67 thought it was a suitable assessment question and 55 that it was not. Fifty seven teachers attempted to solve Question 4, with $26(45.6 \%)$ noticing that the integrand function is not continuous on the interval $[-1,1]$ and $31(54.4 \%)$ incorrectly treating it as a definite integral. Those 31 teachers 'solved' it with a version of $\int_{-1}^{1} \frac{1}{x} d x=\ln |x|_{-1}^{1}=\ln |1|-\ln |-1|=0-0=0 \quad$ (see the examples in Figure 6). Some of these commented that the use of the absolute value would be a problem for students.

$$
\begin{aligned}
& \text { 4. Find the integral } \int_{-1}^{1} d x=\overline{[\ln |x|]_{-1}^{1} \ln 1-\ln |-1|} \\
& \text { 4. Find the integral } \int_{-1}^{1} \frac{1}{x} d x=0
\end{aligned}
$$

Figure 6. Teachers' responses to Question 4 showing misuse of a standard procedure.

Some of the teachers who noticed that the function $y=\frac{1}{x}$ is not defined at $x=0$ still tried to solve the question. They often sketched the graph, and then used this to notice that the 
areas on $[-1,0]$ and $[0,1]$ were the 'same' because of the symmetry and wrongly concluded that the integral is zero. Some typical remarks made by those adopting this stance were: 'By symmetry of areas intuitively the answer is zero'; 'Zero is correct answer - use of symmetry + common sense gives answer as zero'. Basically they relied on intuition or a graphical illustration to answer the question and in this case it failed. Some teachers recognised the issue of continuity (see Figure 7), and a few of these made comments that showed a measure of uncertainty or ambiguity in their conclusions: 'An excellent question - there are two answers - undefined or zero'; 'Numerically gives 0 but undefined because of discontinuity'; 'Comes to 0 this way but actually not defined since $y=\frac{1}{x}$ is discontinuous at $x=0$.'

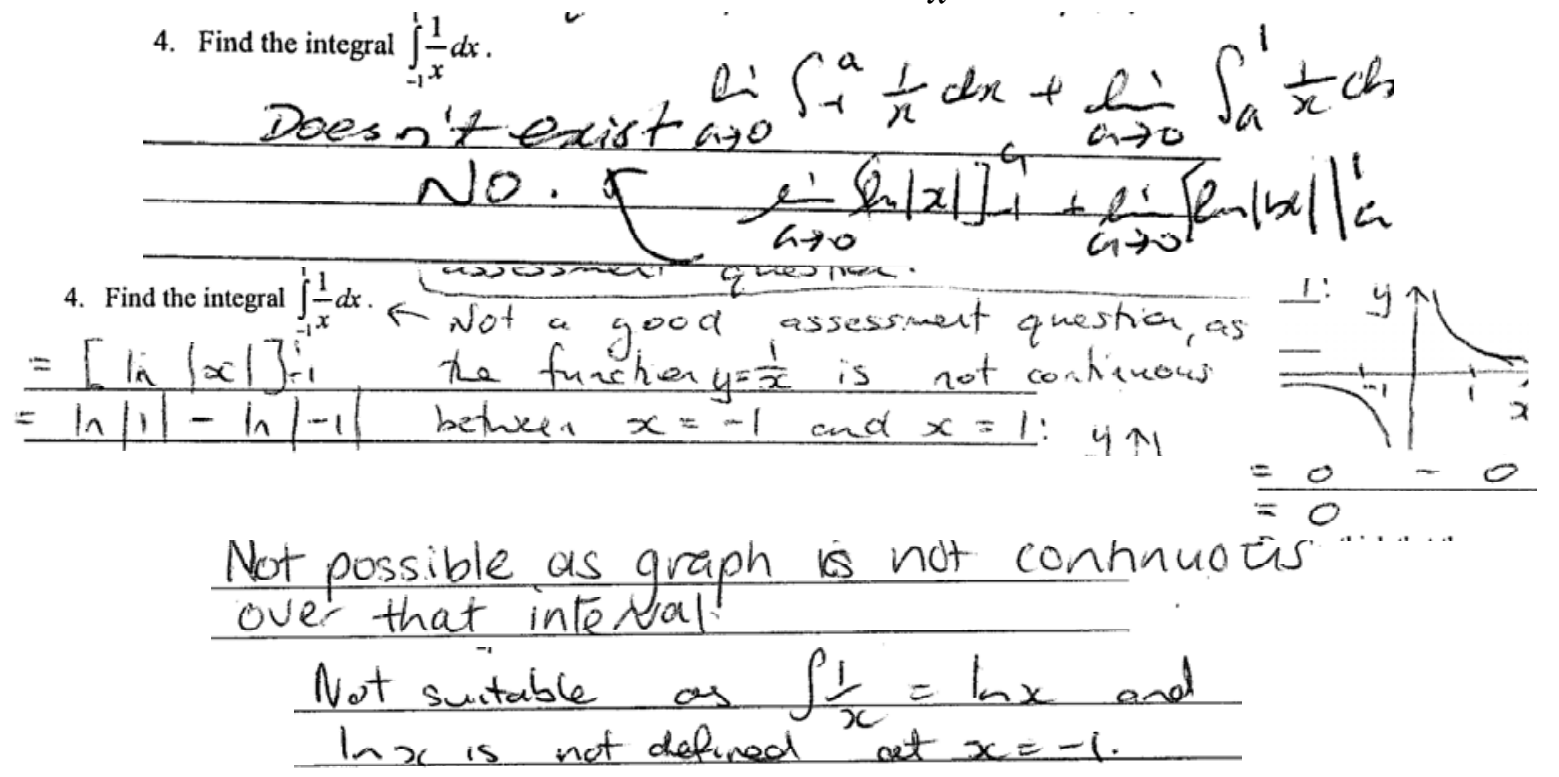

Figure 7. Teachers' responses to Question 4 showing attention paid to continuity or domain.

As for the lecturers, Question 4 should have been a routine question on improper integrals which is often part of a first-year degree calculus course syllabus, and hence we were surprised by some of their answers. Twenty three lecturers responded to Question 4, with 14 $(60.9 \%)$ saying it was a suitable assessment question and $9(39.1 \%)$ that it was not. Eleven lecturers provided their solutions to Question 4 with three $(27.3 \%)$ giving a correct solution and eight $(72.7 \%)$ an incorrect solution. The proportion of incorrect solutions provided by the lecturers was quite alarming and unexpected. Both definite integrals and improper integrals can be common examination questions on the integration topic of a first-year degree calculus course and it is hard to imagine that a lecturer would confuse them. One possible explanation is that some of the lecturers who provided incorrect solutions to Question 4 were teaching at a polytechnic and improper integrals were excluded from their first-year calculus syllabus. Another is that by paying attention to procedures and having a function with a well-known antiderivative they failed to notice the interval contained $x=0$. Figure 8 below illustrates some lecturers' responses to Question 4. 
4. Find the integral $\int_{-1}^{1} \frac{1}{x} d x$.

\section{Trouble at -1 modulus fargotten, but OK.}

$$
\text { 4. Find the integral } \left.\int_{-1}^{1} \frac{1}{x} d x .=\ln x\right]_{-1}^{i}=\operatorname{lon} 1 \cdot \ln (-i)
$$

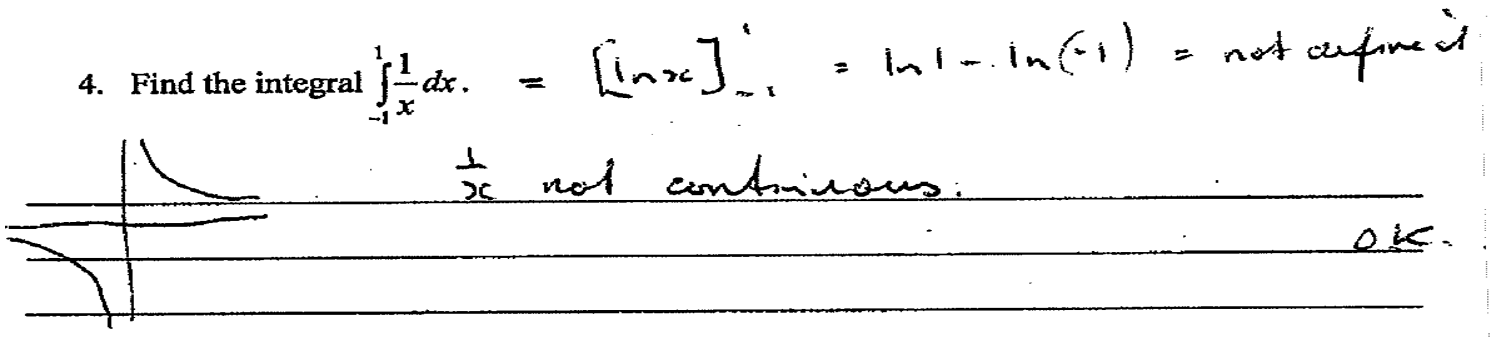

Figure 8. Examples of incorrect lecturers' responses to Question 4.

\section{Conclusions and Recommendations}

The analysis above does raise some issues. The results of the respondents' answers to Questions 3 and 4, which checked their ability to pay attention to the conditions and properties of the mathematical objects involved, showed that both the teachers and lecturers have room for improvement of their discipline of noticing. In Question 3 most of the teachers $(73.5 \%)$ and lecturers $(80 \%)$ failed to notice that the domain of the function was an empty set and provided incorrect solutions. In Question 4 the proportions of incorrect solutions were $54.4 \%$ for the teachers and $72.7 \%$ for the lecturers. Contrary to our expectations this study indicates that the teachers who participated in the study tended to have a better discipline of noticing than the lecturers, although we did not do a detailed statistical analysis of the differences. When evaluating mathematical questions it is important to teach students to pay attention globally and locally, consider conditions, properties and relationships, since all of these aspects are as important to develop as mathematical techniques. It needs to be a natural part of their mathematical culture. The ability to analyse carefully a mathematics question can enhance students' ability to analyse critically other situations outside mathematics. Along with teaching students mathematical procedures, techniques and 'tricks' we should develop their analytical thinking that can also benefit them outside of school or university. Noticing and paying attention to every detail, structure their attention by looking at rather than looking through as Mason [9] suggests is a big aspect of that ability. But to do that both schoolteachers and tertiary lecturers need to have this ability themselves. What influences what we pay attention to? What can help us to attend to important details, etc? Mason $[9, \mathrm{p}$. 11] suggests that we need to be 'developing strategies for directing or focusing attention in a way which is pertinent to the learning', especially towards recognition of properties and reasoning with them. One such strategy is the use of carefully thought-out questions. In his analysis of the teaching process, Schoenfeld [12] describes how teacher beliefs (latterly orientations, including values, etc.) lead to setting goals and the use of appropriate knowledge to guide actions, such as questioning, in pursuit of those goals. Someone who is primarily a symbolic world thinker [4] with a belief that procedural skills are of primary importance may be more likely to pay attention to issues that are relevant to procedures and tend to apply 
these without further thought, rather than considering properties. While other representations, such as graphs, may help structure attention, it may be that emphasising procedures, intuition, 'common sense' or pictures can lead one to miss things it is important to notice, preventing one appreciating the need to check conditions of the rule or theorem, verify properties of the functions involved, or justify the use of a certain technique, etc. This seems to have been the case for a number of our participants. This research suggests that explicit training in the discipline of noticing could be a useful addition to professional development of both school teachers and university lecturers, especially those in the beginning of their career.

\section{Acknowledgements}

We would like to acknowledge a grant from the New Zealand Council for Education Research under the Teaching and Learning Research Initiative that enabled us to conduct this research. An earlier version of this research was presented at PME33 in Thessaloniki, Greece.

\section{References}

[1] A. Smith, Making Mathematics Count, UK: The Stationery Office Limited, 2004.

[2] The National Commission on Mathematics and Science Teaching for the 21st Century, Before it's too late - A report to the nation from The National Commission on Mathematics and Science Teaching for the 21st Century, USA, 2000. Available at http://www2.ed.gov/inits/Math/glenn/report.pdf

[3] M. Clark and M. Lovric, Understanding secondary-tertiary transition in mathematics, Int. J. Math. Educ. Sci. Technol. 40(6) (2009), pp. 755-776.

[4] D. O. Tall, The transition to formal thinking in mathematics, Math. Educ. Res. J. 20(2) (2008), pp. 5-24.

[5] S. Stewart and M. O. J. Thomas, Student learning of basis in linear algebra, in Proceedings of the Joint Conference of PME 32 and PME-NA, O. Figueras, J. L. Cortina, S. Alatorre, T. Rojano, A. Sepulveda, eds., Morelia, Mexico, 2008, vol. 4, pp. 281-288.

[6] J. Novotna and M. Hoch, How structure sense for algebraic expressions or equations is related to structure sense for abstract algebra, Math. Educ. Res. J. 20(2) (2008), pp. 93-104.

[7] D. L. Ball, H. C. Hill, and H. Bass, Who knows mathematics well enough to teach third grade, and how can we decide? American Educator, Fall (2005), pp. 14-22 and 43-46.

[8] H. Hill and D. L. Ball, Learning mathematics for teaching: Results from California's mathematics professional development institutes. J. Res. Math. Edu. 35(5) (2004), pp. 330-351.

[9] J. Mason, Doing $\neq$ construing and doing + discussing $\neq$ learning: The importance of the structure of attention, in Proceedings of the $10^{\text {th }}$ International Congress on Mathematics Education (ICME-10), Copenhagen, Denmark, 2008. Available at http://www.icme10.dk/proceedings/pages/regular_pdf/RL_John_Mason.pdf.

[10] J. Mason, Asking mathematical questions mathematically. Int. J. Math. Educ. Sci. Technol. 31(1) (2000), pp. 97-111.

[11] J. Mason and M. Spence, Beyond mere knowledge of mathematics: The importance of knowing-to act in the moment. Educ. Stud. Math. 38 (1999), pp. 135-161.

[12] A. H. Schoenfeld, Models of the teaching process. Journal of Mathematical Behaviour 18(3) (1999), pp. 243-261. 